MATEC Web of Conferences 3, 01024 (2013)

DOI: $10.1051 /$ matecconf/20130301024

(0) Owned by the authors, published by EDP Sciences, 2013

\title{
Flow calorimetry: a technique for caracterisation of liquid-liquid and liquid-gas systems
}

\author{
M.R. Simond ${ }^{1,2}$, K. Ballerat-Busserolles ${ }^{1,2}$, Y. Coulier ${ }^{1,2}$, and J-Y. Coxam ${ }^{1,2}$ \\ ${ }^{1}$ Clermont Université, Université Blaise Pascal, Institut de Chimie de Clermont-Ferrand, B.P 80026, F-63171 Aubière, \\ France \\ ${ }^{2}$ CNRS, UMR 6296, ICCF-TIM, BP 80026, F-63171 Aubière, France
}

\section{Introduction}

Flow calorimetric techniques have been developed for thermodynamic studies of fluid systems. The systems investigated are mainly of interest for the development of industrial processes for $\mathrm{CO}_{2}$ capture in post combustion industrial effluents. The explored systems are complex because of numerous components to be considered. A realistic or rigorous thermodynamic representation must take into account non-ideality. Then many interaction parameters need to be determined to adjust theoretical model and then, wide and consistent experimental data are required.

The capture process particularly studied in our lab is based on chemical and physical dissolution of carbon dioxide in aqueous solutions of amine. In open literature, most of experimental data for $\left\{\mathrm{CO}_{2}+\right.$ amine + water $\}$ systems give the total $\mathrm{CO}_{2}$ in solution as function of total pressure and temperature. The models developed with such equilibrium data can correlate gas solubility. However it have been shown [1] that they cannot correctly predict enthalpic data. The validity of a model should be checked out by measurements of enthalpy of solution of $\mathrm{CO}_{2}$ [1]. This enthalpy is strongly dependent on the mechanism of absorption and then to the phase composition reached at equilibrium. Nevertheless, literature data for enthalpy is relatively scarce.

Our laboratory develops calorimetric techniques for the determination of enthalpy of mixing adapted to study $\{$ liquid + liquid $\}$ or $\{$ liquid + gas $\}$ systems. The description and principles of the different techniques are illustrated by examples in relation with the thematic of $\mathrm{CO}_{2}$ capture by dissolution in aqueous solutions of amines.

This paper will report examples and emphasise the limitations and difficulties associated to measurements at super ambient conditions.

\section{Experimental}

The calorimetric technique used to measure heat of mixing is schematically represented in figure 1 . The heat of mixing of two fluids is determined in dynamic mode [2]. The calorimetric signal is a differential heat flux exchange with a calorimetric block during mixing. The mixing is performed in a customized "mixing flow unit" specially designed for Setaram heat conduction differential calorimeters (C80 or BT215). The fluids flow by use of two high-pressure syringe pumps. The two fluids encounter at a mixing point and then the mixture flow in a tubing coiled in good thermal contact with the inner wall of the confinement cylinder (mixing cell). Preheaters are placed along the flow line to set up the temperature of entering fluids at same value as that of the mixing cell, at temperature in equilibrium with the calorimetric block. The number and temperature of preheaters have to be adjusted in order to control the temperature of incoming fluids regardless injection flow rate.

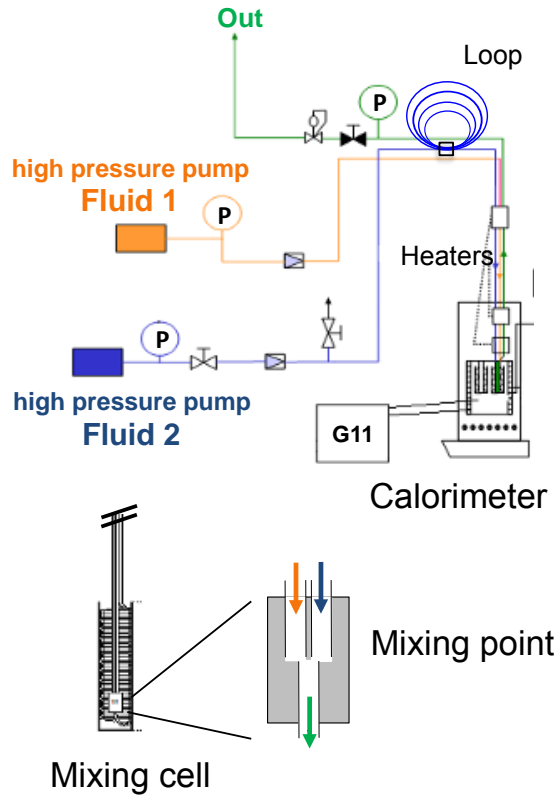

Figure 1. Schematic representation of the calorimetric line. 
In order to preserve high pressure pumps from corrosion, an additional injection loop is inserted between the pumps and the mixing cell. The pumps are currently filled with water, and used to "push" corrosive fluids. The experiments are carried out at constant pressure. The pressure is measured at the input and output of the mixing cell to detect any problem of over pressure, especially when working with viscous fluids. Experiments are carried out at different mixture compositions by changing flow rate ratio of the high pressure pumps. The signal of the thermopile surrounding the mixing cell is converted into heat flux using a calibration constant determined by joule effect. The value of this constant is validated by chemical calibration running experiments with reference systems such as $\{$ ethanol + water $\}$. Example of chemical calibration is presented in figure 2. The enthalpies of mixing of ethanol with water at $333.15 \mathrm{~K}$ were compared with reference values obtained from the data recommended by Ott et al.[3].

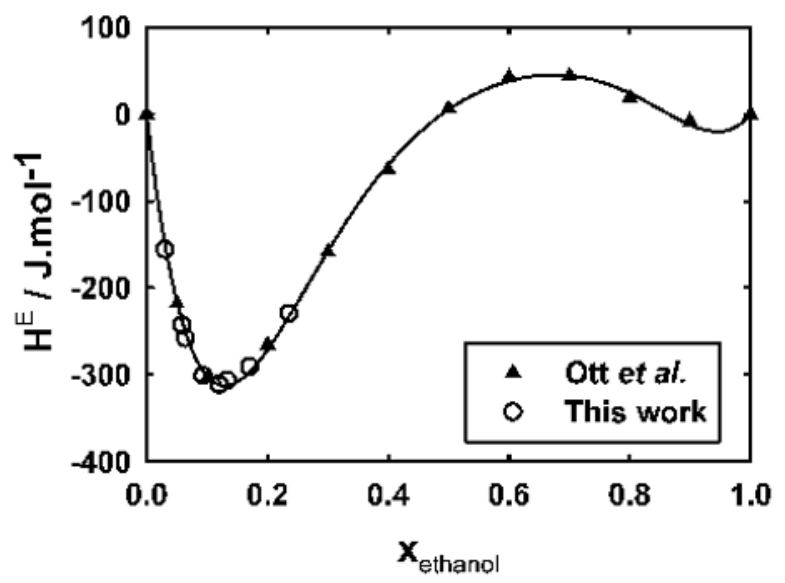

Figure 2. Chemical calibration using $\{$ water + ethanol $\}$ at $333.15 \mathrm{~K}$.

\section{Results}

The main objective of a thermodynamic representation of the ternary system $\left\{\mathrm{CO}_{2}+\right.$ amine + water $\}$ is to give tools to correlate and predict absorption properties. In addition it can give information on the mechanisms involved in the gas dissolution. $\mathrm{CO}_{2}$ dissolution in aqueous solutions of amines is complex. It includes many chemical reactions, leading to formation of ionic compounds like protonated amine, carbonate, bicarbonate or carbamates. The thermodynamic representation of $\left\{\mathrm{CO}_{2}+\right.$ amine + water $\}$ system will then necessitate investigations on subsystems such as $\left\{\mathrm{CO}_{2}+\right.$ amine + water $\}, \quad$ amine + water $\} \quad$ and \{amine + acid + water $\}$.

Flow calorimetric technique is first used to provide essential energetic information on the absorption. As an example, enthalpies of solution of carbon dioxide in aqueous solutions of $\mathrm{N}$-methyldiethanolamine (MDEA), at $322.15 \mathrm{~K}$ and $1 \mathrm{MPa}$, are reported in figure 3 . The enthalpy is expressed per mole of carbon dioxide mixed with the amine solution $\left(-\Delta_{\mathrm{s}} H^{*}\right)$, and plotted versus loading charge $\alpha$ (moles of $\mathrm{CO}_{2}$ per mole of amine). This enthalpy $\left(-\Delta_{s} H^{*}\right)$ appears to be constant within experimental uncertainty for $\alpha$ below 0.5 . This behaviour is currently observed and the average value on the plateau (figure 3 ) is used to compare the energy of absorption as function of the amine or the temperature. However, loading charge $\alpha$ below 0.05 can hardly be investigated by our technique. The enthalpy behaviour at low loading charges predicted by models (figure 4) was not checked. The enthalpy of solution can also be expressed per mole of amine $\left(-\Delta_{s} H^{\star}\right)$. In this case, the enthalpy curve shows two regions. The linear increase of enthalpy (open triangles on figure 3 ) corresponds to the dissolution of an increasing quantity of $\mathrm{CO}_{2}$. The plateau is related to the saturation of the absorbent solution. Then solubility limit $\left(\alpha^{*}\right)$ can be graphically estimated as the first point of the plateau (intersection of the two regions).

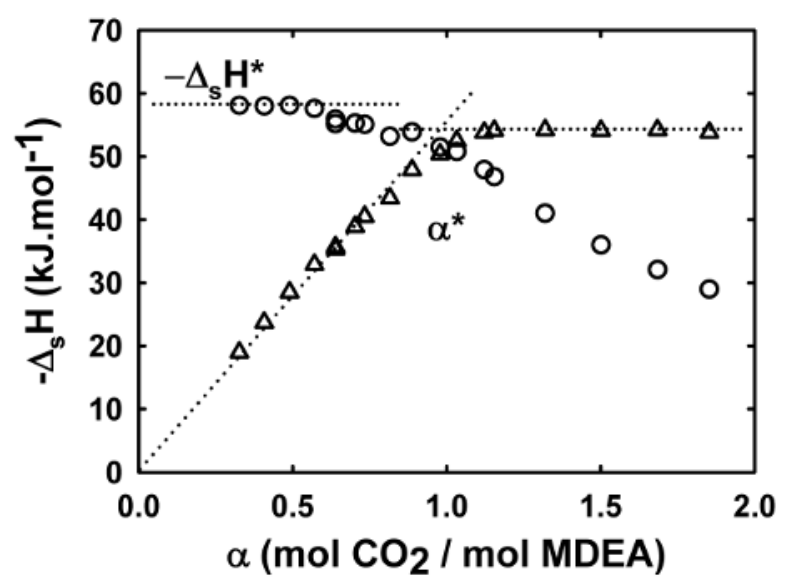

Figure 3. Enthalpy of absorption of carbon dioxide in solution of MDEA at $322.15 \mathrm{~K}$ and $1 \mathrm{MPa}$.

The uncertainty on the graphical determination will depend on the mechanism of dissolution. For loading charge above 1 , significant physical dissolution of $\mathrm{CO}_{2}$ is observed. Then a slight decrease in the slope of the enthalpy curve before reaching the plateau is perceived. Then the estimation of intersection between unsaturated and saturated domains is more difficult and the uncertainty on the limit of gas solubility can reach $10 \%$. This limit of gas solubility is a way to validate the calorimetric measurement. In another hand, to be sure that all the energy of mixing is dissipated inside the mixing cell, experiments are carried out at different flow rates. 


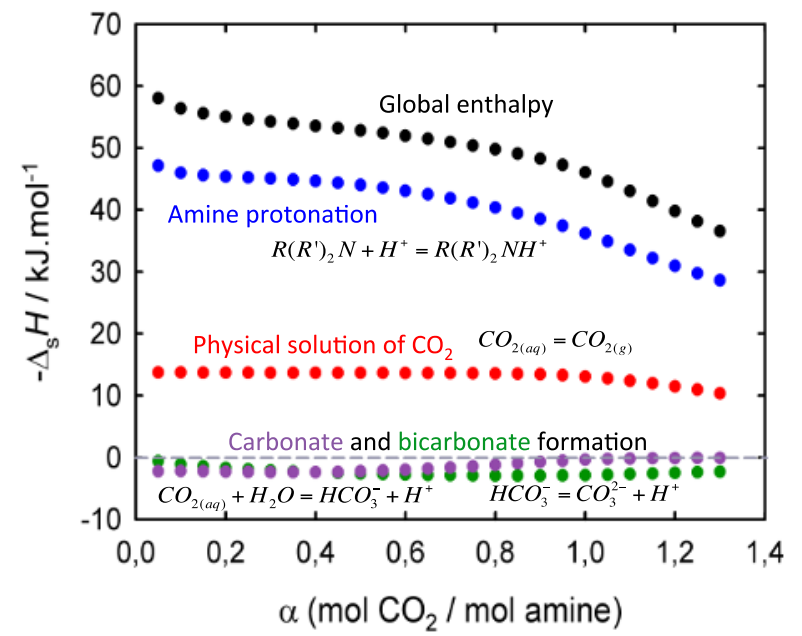

Figure 4. Solution enthalpies of $\mathrm{CO}_{2}$ per mole of $\mathrm{CO}_{2}$ in MDEA and different enthalpic contributions versus loading charge using thermodynamic model [1].

To better understand the mechanism of absorption, the total enthalpy could be split in different contributions. Each contribution is related to one chemical or physical reaction, occurring during dissolution. Thermodynamic models (Arcis et al. [1]) can predict those terms. As shown in figure 4 , the enthalpy of absorption, in tertiary amine solutions, is mainly impacted by the enthalpy of protonation. Theoretical enthalpic values are derived from a temperature correlation function of the amine protonation constant. $\mathrm{pKa}$ values for amines [4] can be obtained by potentiometric titration technique, as function of temperature. As an example, protonation constant for MDEA are reported in figure 6 , for temperature ranges from $298 \mathrm{~K}$ to $373 \mathrm{~K}$. Potentiometric measurements at high temperature are however difficult and calorimetric technique can be used to determine the temperature behaviour of $\mathrm{pKa}$, by direct measurement of the enthalpy of protonation. Our flow technique was then used to test consistency of existing potentiometric data and to extend data at higher temperatures.

For example, in case of MDEA, two correlations of $\mathrm{pKa}$ with temperature are obtained from different potentiometric methodologies. Two sets of data can be distinguished in literature (figure 6): one serie from Littel and co-workers [5] that differs slightly from a second set, that includes Perez-Salado Kamps and Maurer [6] and our experimental results [4]. Thus, enthalpies of protonation were determined at different conditions of pressure $(0.5$ and $1.1 \mathrm{MPa})$ and composition $(\mathrm{HCl}$ and amine solutions) using our flow calorimetric technique. In order to test consistency of protonation constant in figure 6 , the temperature dependence of $\mathrm{pKa}$ was calculated from our enthalpies, using Van't Hoff equation. The reference $\mathrm{pKa}$ value was measured at 298.15 K, using potentiometric method.

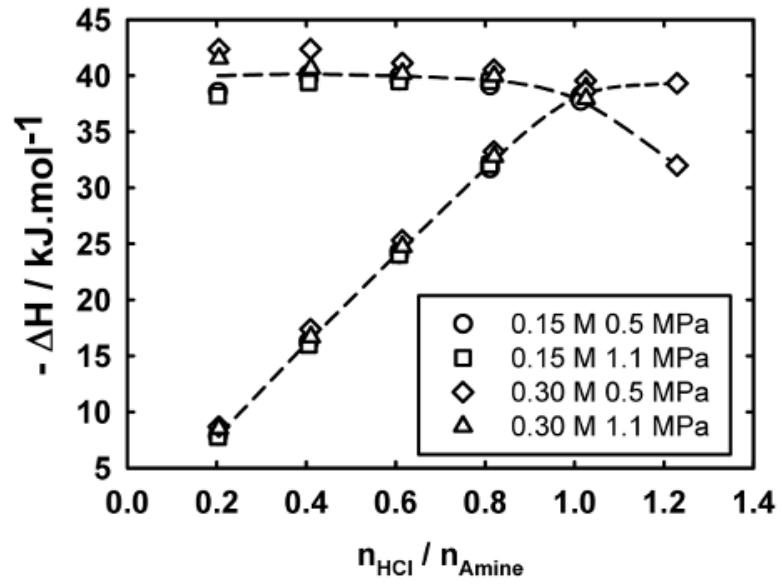

Figure 5. Protonation enthalpy of MDEA titrated by $\mathrm{HCl}$ at different conditions of concentration and pressure.

Figure 5 represents the enthalpy of protonation obtained at different chemical advancements of reaction. The behavior is similar to the one obtained for gas dissolution. The enthalpy expressed per mole of amine increases linearly up to neutralization while the enthalpy expressed per mole of $\mathrm{HCl}$ remains quite constant until neutralization, and then decreases. The $\mathrm{pKa}$ was calculated for each pressure, concentration and chemical advancement of reaction.

No pressure or concentration effects were observed; the $\mathrm{pKa}$ differences were within the experimental uncertainty. Then we reported in figure 6 average values of protonation constants at temperatures from 313.15 to 373.15 K. Figure 6 shows very good consistency between potentiometric and calorimetric results determined in the laboratory, and good agreement is also observed with values of Perez-Salado Kamps and Maurer [6].

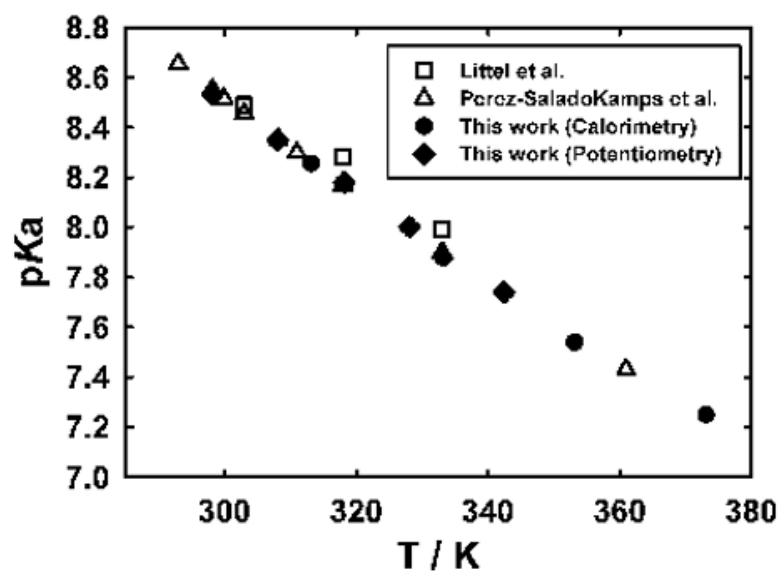

Figure 6. $\mathrm{p} K \mathrm{a}$ vs temperature of MDEA.

Finally, our calorimetric technique was more recently used to investigate a new class of amines called "demixing amine". The specificity of aqueous solutions is a partial miscibility. These so called "thermomorphic" biphasic amine solvents are potential absorbent for breakthrough technologies of $\mathrm{CO}_{2}$ capture [7]. The phase separation will be induced at the output of the absorber. Then, to regenerate the absorbent in a process working on 
absorption-desorption cycles, only the aqueous phase containing $\mathrm{CO}_{2}$ will be treated to separate the gas. The energy to separate $\mathrm{CO}_{2}$ from the solution will be globally lowered, compared to classical $\mathrm{CO}_{2}$ capture, by decreasing the total volume of solution to be treated. In addition to gas absorption, the phase separation has to be characterized. Flow calorimetric method has been applied to study binary systems \{amine + water $\}$. The enthalpy of mixing water with amine, or, excess enthalpy, is easily measured as function of temperature and pressure. Excess enthalpies of $\{\mathrm{N}$-methylpiperidine + water $\}$ system at 303.15 and $333.15 \mathrm{~K}$ and $0.5 \mathrm{MPa}$ are reported in figure 7.

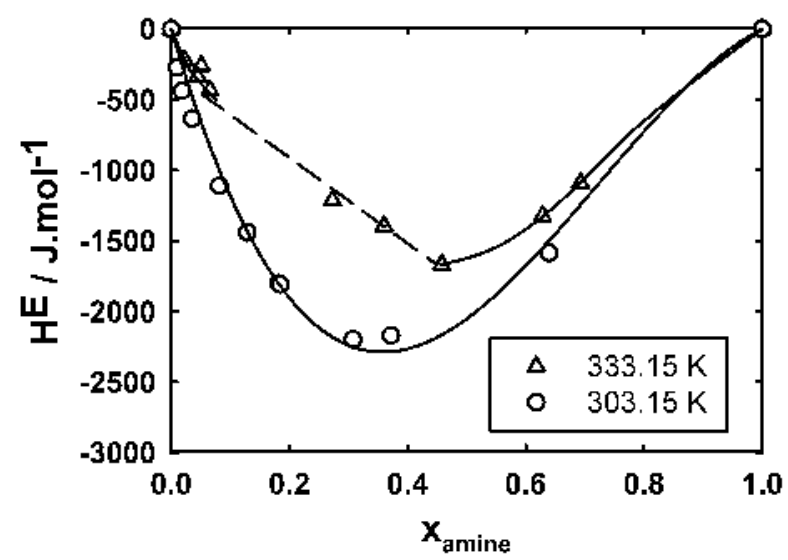

Figure 7. Excess enthalpy of $\{\mathrm{N}$-methypipéridine + water $\}$ sytem at $303.15 \mathrm{~K}$ and $333.15 \mathrm{~K}$ and $0.5 \mathrm{MPa}$.

At $303.15 \mathrm{~K}$ the system show total miscibility and the enthalpies can be fitted using Redlich-Kister equation. At $333.15 \mathrm{~K}$ we can note a linear behaviour for molar fractions $x_{\text {amine }}$ between 0.04 and 0.45. This domain corresponds to equilibrium between two immiscible liquid phases. The compositions at the limits of the linear region are corresponding to the composition of two immiscible phases in equilibrium at this temperature. It was observe that they are in good agreement with the phase diagram obtained by Coulier et al. [8]. This results represent a validation and test for the enthalpy measurements. The main difficulty in the measurement of enthalpy of mixing of two liquids is the viscosity. As viscous amine does not mix easily with water, and the fluids flow rates have to be carefully selected.

\section{Conclusion}

Flow calorimetric technique is of great interest for the thermodynamic representation of the absorption of carbon dioxide in aqueous solutions of amine. The technique can be used to study \{liquid + liquid $\}$ or $\{$ gas + liquid $\}$ related systems. However, working in dynamic mode supposes that the fluids mix correctly in the mixing cell, with fast kinetic. In each case, the validity of enthalpy data can be checked by testing the consistency with other properties. For example, the measurements of enthalpy of solution of carbon dioxide in aqueous solution of amine can be tested, by comparing the limits of gas solubility.

\section{References}

1. H. Arcis, L. Rodier, K. Ballerat-Busserolles, J.-Y. Coxam, J. Chem. Thermodyn. 41783 (2009)

2. H. Arcis, K. Ballerat-Busserolles, L. Rodier, J.-Y. Coxam, J. Chem. Eng. Data 563351 (2011)

3. J.B. Ott, G.V. Cornett, C.E. Stouffer, B.F. Woodfield, C. Guanquan, J.J. Christensen, J. Chem. Thermodyn. 18867 (1986)

4. M. Simond, K. Ballerat-Busserolles, Y. Coulier, L. Rodier, J.-Y. Coxam, J. Solution Chem. 41130 (2012)

5. R.J. Littel, M. Bos, G.J. Knoop, J. Chem. Eng. Data 35276 (1990)

6. Á. Pérez-Salado Kamps, G. Maurer, J. Chem. Eng. Data 411505 (1996)

7. L. Raynal, P.-A. Bouillon, A. Gomez, P. Broutin, Chemical Engineering Journal 171742 (2011)

8. Y. Coulier, K. Ballerat-Busserolles, L. Rodier, J.-Y. Coxam, Fluid Phase Equilibr. 296206 (2010) 